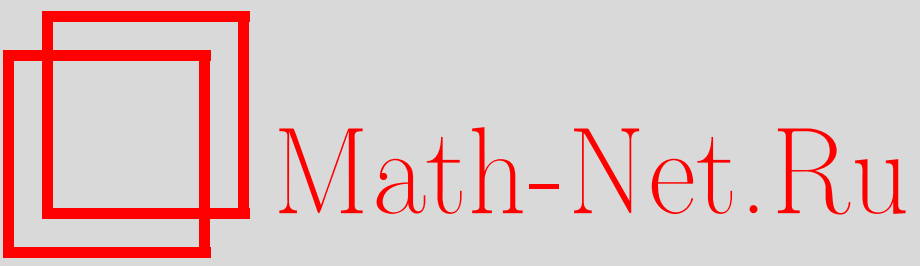

О. А. Ковалева, Об одном свойстве индекса нелокальных эллиптических задач, УМH, 1999, том 54, выпуск 4, 173-174

DOI: https://doi.org/10.4213/rm189

Использование Общероссийского математического портала Math-Net.Ru подразумевает, что вы прочитали и согласны с пользовательским соглашением

http://www.mathnet.ru/rus/agreement

Параметры загрузки:

IP: 34.227 .88 .159

26 апреля 2023 г., $16: 39: 32$ 


\title{
ОБ ОДНОМ СВОЙСТВЕ ИНДЕКСА НЕЛОКАЛЬНЫХ ЭЛЛИПТИЧЕСКИХ ЗАДАЧ
}

\author{
О. А. КовАЛЕВА
}

Рассматриваются эллиптические уравнения порядка $2 m$ в ограниченной области $Q \subset \mathbb{R}^{n}$ с границей $\partial Q$ и нелокальными условиями, связывающими следы решения и его производных на $(n-1)$-мерных гладких многообразиях $\Gamma_{i}\left(\bigcup_{i} \bar{\Gamma}_{i}=\partial Q\right)$ с их значениями на некотором компакте $\Omega \subset \bar{Q}$. В случае $\Omega \cap \mathscr{K}_{1}=\varnothing$ мы сформулируем теоремы об устойчивости индекса нелокальных задач в весовых пространствах $V_{p, a}^{k+2 m}(Q)$, где $\mathscr{K}_{1}=\bigcup_{j}\left(\bar{\Gamma}_{j} \backslash \Gamma_{j}\right)$. Для $p=2$ эти результаты были получены в [1]. Отметим, что вопрос о сохранении нулевого индекса для некоторых классов нелокальных возмущений задачи Дирихле для эллиптических уравнений второго порядка в других функциональных пространствах изучался в [2], [3].

Пусть $Q \subset \mathbb{R}^{n}$ - ограниченная область с границей $\partial Q=\bigcup_{i} \bar{\Gamma}_{i}\left(i=1, \ldots, N_{0}\right)$, где $\Gamma_{i}$ суть $(n-1)$-мерные многообразия класса $C^{\infty}$, открытые в топологии $\partial Q$. Будем предполагать, что некоторая окрестность каждой точки $g \in \partial Q \backslash \bigcup_{i} \Gamma_{i}$ диффеоморфна двугранному углу $\mathscr{D}_{g}=$ $\left\{x=(y, z) \in \mathbb{R}^{n}:|\varphi|<b, z \in \mathbb{R}^{n-2}\right\}, n \geqslant 3 ;$ или плоскому углу $K_{g}=\left\{x=y \in \mathbb{R}^{2}:|\varphi|<b\right\}$, $n=2$. Здесь $0<b<\pi,(r, \varphi)$ - полярные координаты на плоскости $\{y\}$. Введем множество $\mathscr{K}$ по формуле $\mathscr{K}=\mathscr{K}_{1} \cup \mathscr{K}_{2} \cup \mathscr{K}_{3}$, где $\mathscr{K}_{1}=\partial Q \backslash \bigcup_{i} \Gamma_{i}=\bigcup_{\nu} \mathscr{K}_{1 \nu}, \mathscr{K}_{2}=\bigcup_{\nu} \mathscr{K}_{2 \nu} \subset \bigcup_{i} \Gamma_{i}, \mathscr{K}_{3}=$ $\bigcup_{\nu} \mathscr{K}_{3 \nu} \subset Q ; \mathscr{K}_{j \nu}\left(j=1,2,3 ; \nu=1, \ldots, N_{j}\right)$ - непересекающиеся замкнутые $(n-2)$-мерные многообразия класса $C^{\infty}$. В случае $n=2 \mathscr{K}_{j \nu}$ - изолированные точки и $N_{0}=N_{1}$.

Пусть в $Q$ существует функция $\rho=\rho(x) \in C^{\infty}\left(\mathbb{R}^{n} \backslash \mathscr{K}\right)$, значения которой в некоторой окрестности множества $\mathscr{K}$ совпадают с расстоянием от точки $x \in Q$ до $\mathscr{K}$, а вне этой окрестности $\rho(x) \geqslant c>0$. Введем пространство $V_{p, a}^{k}(Q)$ как пополнение множества $\stackrel{\circ}{C}(\bar{Q} \backslash \mathscr{K})$ по норме

$$
\|u\|_{V_{p, a}^{k}(Q)}=\left\{\sum_{|\alpha| \leqslant k} \int_{Q} \rho^{p(a-k+|\alpha|)}\left|D^{\alpha} u\right|^{p} d x\right\}^{1 / p},
$$

где $a \in \mathbb{R}, \stackrel{\circ}{C^{\infty}}(\bar{Q} \backslash \mathscr{K})$ - множество бесконечно диффференцируемых функций с компактными носителями в $\bar{Q} \backslash \mathscr{K}$. Через $V_{p, a}^{k-1 / p}(\Gamma)$ обозначим пространство следов на $(n-1)$-мерном гладком многообразии $\Gamma \subset \bar{Q}$ с нормой

$$
\|\psi\|_{V_{p, a}^{k-1 / p}(\Gamma)}=\inf \|u\|_{V_{p, a}^{k}(Q)} \quad\left(u \in V_{p, a}^{k}(Q):\left.u\right|_{\Gamma}=\psi\right) .
$$

Рассмотрим задачу

$$
\text { (2) } \quad B_{i \mu} u=\left.B_{i \mu}^{0} u\right|_{\Gamma_{i}}+B_{i \mu}^{1} u+B_{i \mu}^{2} u=f_{i \mu}(x) \quad\left(x \in \Gamma_{i} ; i=1, \ldots, N_{0} ; \mu=1, \ldots, m\right) \text {. }
$$

Здесь

$$
\begin{gathered}
A_{0}(x, D)=\sum_{|\alpha|=2 m} a_{\alpha}(x) D^{\alpha}, \quad B_{i \mu}^{0}(x, D)=\sum_{|\alpha|=m_{i \mu}} b_{i \mu 0 \alpha}(x) D^{\alpha} ; \\
a_{\alpha}, b_{i \mu 0 \alpha} \in C^{\infty}\left(\mathbb{R}^{n}\right), \quad f=\left\{f_{0}, f_{i \mu}\right\} \in \mathcal{V}_{p, a}^{k}(Q, \partial Q)=V_{p, a}^{k}(Q) \times \prod_{i} \prod_{\mu} V_{p, a}^{k+2 m-m_{i \mu}-\frac{1}{p}}\left(\Gamma_{i}\right)
\end{gathered}
$$

- комплекснозначные функции.

Предположим, что выполняются следующие условия.

1. Оператор $A_{0}(x, D)$ - правильно эллиптический для всех $x \in \bar{Q}$.

2. Система операторов $B_{i \mu}^{0}(x, D), \mu=1, \ldots, m$, удовлетворяет условию Лопатинского по отношению к оператору $A_{0}(x, D)$ для всех $i=1, \ldots, N_{0}$ и $x \in \bar{\Gamma}_{i}$. 
3. Если $n \geqslant 3$, то $m_{i \mu} \leqslant 2 m-1$ и система операторов $B_{i \mu}^{0}(x, D), \mu=1, \ldots, m$, является нормальной для всех $x \in \bar{\Gamma}_{i}$ и $i=1, \ldots, N_{0}$.

4. Операторы

$$
\begin{gathered}
A_{1}: V_{p, a}^{k+2 m-1}(Q) \rightarrow V_{p, a}^{k}(Q), \\
B_{i \mu}^{1}: V_{p, a}^{k+2 m-1}(Q) \rightarrow V_{p, a}^{k+2 m-m_{i \mu}-\frac{1}{p}}\left(\Gamma_{i}\right), \\
B_{i \mu}^{2}: V_{p, a}^{k+2 m}(Q) \rightarrow V_{p, a}^{k+2 m-m_{i \mu}-\frac{1}{p}}\left(\Gamma_{i}\right)
\end{gathered}
$$

- ограниченные, и существуют числа $\varepsilon, \sigma$ такие, что для всех $u \in V_{p, a}^{k+2 m}(Q)$

$$
\begin{gathered}
\left\|B_{i \mu}^{2} u\right\|_{V_{p, a}^{k+2 m-m_{i \mu}-\frac{1}{p}}\left(\Gamma_{i}\right)}^{k} \leqslant c_{1}\|u\|_{V_{p, a}^{k+2 m}}\left(Q \backslash S_{2 \varepsilon}\left(\mathscr{K}_{1}\right)\right) \\
\left\|B_{i \mu}^{2} u\right\|_{V_{p, a}^{k+2 m-m_{i \mu}-\frac{1}{p}}\left(\Gamma_{i} \backslash S_{\varepsilon}\left(\mathscr{K}_{1}\right)\right)} \leqslant c_{2}\|u\|_{V_{p, a}^{k+2 m}\left(Q_{\sigma}\right)},
\end{gathered}
$$

где $S_{\delta}\left(\mathscr{K}_{1}\right)=\left\{x \in \mathbb{R}^{n}: \rho\left(x, \mathscr{K}_{1}\right) \leqslant \delta\right\}$.

Определим операторы $\mathscr{L}_{0}, \mathscr{L}: V_{p, a}^{k+2 m}(Q) \rightarrow \mathscr{V}_{p, a}^{k}(Q, \partial Q)$ по следуюшим формулам $\mathscr{L}_{0}=$ $\left\{A_{0} u,\left.B_{i \mu}^{0} u\right|_{\Gamma_{i}}\right\}, \mathscr{L} u=\left\{A u, B_{i \mu} u\right\}$.

Теорема 1. Пусть въполняются условия 1-4 и оператор $\mathscr{L}_{0}-$ фредгольмов. Тогда

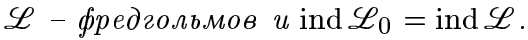

Отметим, что достаточные условия фредгольмовости оператора $\mathscr{L}_{0}$ для плоских областей с угловыми точками и пространственных областей с коническими точками были получены в [4]. Используя методы, предложенные в [5], можно получить достаточные условия фредгольмовости оператора $\mathscr{L}_{0}$ в нашем случае.

ПРимеР 1. Рассмотрим краевую задачу

$$
\begin{gathered}
\Delta u=f_{0}(x) \quad(x \in Q \backslash \mathscr{K}), \\
\left.u(x)\right|_{\Gamma_{1}}+\left.\alpha(x) u(\omega(x))\right|_{\Gamma_{1}}=f_{1}(x) \quad\left(x \in \Gamma_{1}\right), \\
\left.u(x)\right|_{\Gamma_{2}}=f_{2}(x) \quad\left(x \in \Gamma_{2}\right) .
\end{gathered}
$$

Здесь область $Q \in \mathbb{R}^{2}$ с границей $\partial Q \in C^{\infty}, \partial Q=\Gamma_{1} \cup \Gamma_{2} \cup \mathscr{K}_{1} ; \Gamma_{i}$ - открытые дуги $(i=1,2)$, множество $\mathscr{K}_{1}$ состоит из точек $g_{1}, g_{2}$, которые являются концами дуг $\Gamma_{i} ; \omega-$ невырожденное бесконечно дифференцируемое преобразование, отображающее некоторую окрестность $\gamma$ дуги $\bar{\Gamma}_{1}$ на $\omega(\gamma)$ так, что $\omega\left(\Gamma_{1}\right) \subset Q$ и $\omega\left(\Gamma_{1}\right) \cap \mathscr{K}_{1}=\varnothing ; \alpha \in C^{\infty}\left(\mathbb{R}^{n}\right)$ - комплекснозначная функция. Множество $\mathscr{K}=\mathscr{K}_{1} \cup \omega\left(\mathscr{K}_{1}\right) \cup \omega\left(\omega\left(\mathscr{K}_{1}\right) \cap \Gamma_{1}\right)$. Очевидно, из условия $\omega\left(\bar{\Gamma}_{1}\right) \cap \mathscr{K}_{1}=\varnothing$ следует выполнение неравенств (3), (4), в которых $B_{11}^{2} u=\left.\alpha(x) u(\omega(x))\right|_{\Gamma_{1}}, m_{i \mu}=0, m=1$, $2 \varepsilon<\rho\left(\omega\left(\bar{\Gamma}_{1}\right), \mathscr{K}_{1}\right), \sigma<\rho\left(\omega\left(\bar{\Gamma}_{1} \backslash S_{\varepsilon}\left(\mathscr{K}_{1}\right)\right), \partial Q\right)$.

Автор благодарит А. Л. Скубачевского за постановку задачи и постоянное внимание к работе.

\section{СПИСОК ЛИТЕРАТУРЫ}

[1] Skubachevskii A. L. // J. Math. Anal. Appl. 1991. V. 160. № 2. P. 323-341. [2] Кишкис К. Ю. // Дифференц. уравнения. 1988. Т. 24. №1. С. 105-110. [3] Гущин А. К., Михайлов В. П. // Матем. сб. 1994. Т. 185. № 1. С. 121-160. [4] Кондратьев В. А. // Труды ММО. 1967. Т. 16. С. 209-292. [5] Мазья В. Г., Пламеневский Б. А. // Труды ММО. 1978. Т. 37. C. $49-93$. 